\title{
Pragmatic tuberculosis prevention policies for primary care in low- and middle-income countries
}

\author{
To the Editor:
}

Despite being a curable and preventable disease, tuberculosis is the leading cause of death from infection worldwide and is one of the top 10 causes of death from any cause, including in children [1]. Incidence is at best barely declining, increasing in some countries, and some prevalence surveys in high-burden countries have demonstrated a significantly higher tuberculosis burden than estimated [1]. Between a quarter and a third of the world's population is estimated to be infected with tuberculosis, representing a vast reservoir from which new cases arise [1]. Many of these people are never identified or tested, and even among those who are, only a small proportion receive preventive treatment [2]. Interventions that aim to increase preventive treatment uptake and completion are likely to have a greater impact on tuberculosis control and elimination than those focussing on improving completion of treatment by patients [3].

In their recent article in the European Respiratory Journal, ACUÑA-VILLAORDUÑa et al. [4] demonstrate once again that contacts of patients with tuberculosis are at increased risk of tuberculosis infection and disease. In this study of contacts of any age from a setting with low tuberculosis incidence (38 per 100000) and HIV prevalence ( $<1 \%$ of the general population), 507 (71\%) out of 710 contacts had tuberculosis infection (defined by a positive tuberculin skin test (TST) or interferon- $\gamma$ release assay (IGRA)). Over 5 years of follow-up, $36(4.1 \%)$ out of 894 had co-prevalent or incident tuberculosis disease, equating to an overall incidence of 541 per 100000 person-years: at least 14 times that of the general population. Despite this markedly elevated risk, only a minority (16\%) of contacts received preventive treatment, reflecting the low rates of uptake seen globally [2]. Whilst guidelines for preventive treatment in low- and middle-income countries (LMICs) focus principally on children aged $<5$ years due to the high risk of primary progressive disease and death in this age group, it should be highlighted that in this study 21 (91\%) out of 23 of the incident tuberculosis cases occurred in contacts aged $\geqslant 15$ years, of whom only $10 \%$ received preventive treatment [4].

In LMICs, the great majority of patients with tuberculosis and their contacts are managed in primary care. Healthcare workers consistently face the triple challenges of insufficient human resources, scarce or inadequate material resources, and a large, heterogeneous population presenting with complex medical and social syndromes. AcuÑa-Villaorduña et al. [4] describe how the absence of clear definitions of contacts at highest risk of tuberculosis prohibits effective implementation of contact investigation and preventive treatment in these settings. As a potential solution, they used an adapted Mandalakas score to predict tuberculosis infection and disease [5]. Although higher scores, reflecting increased exposure to potentially more infectious index cases, were associated with an increased risk of both infection and disease in contacts, the overall performance of the score for predicting infection was poor. Notably, the score's performance for predicting tuberculosis disease, the rarer but more important outcome, was substantially improved. In their discussion and conclusion, the authors suggest that a laboratory biomarker with moderate accuracy to predict tuberculosis disease is urgently needed to guide targeted preventive treatment for contacts at highest risk. Although such a biomarker would evidently be welcome, we believe that rather than better, expensive, commercial laboratory tests, more pragmatic and simple healthcare policies for contact investigation and preventive treatment are needed that reflect the realities of the primary care centres in LMICs where they are implemented.

@ERSpublications

Improving tuberculosis prevention in low- and middle-income countries requires scaling up of simple policies, not better laboratory tests http://ow.ly/VPLL30iPiZA

Cite this article as: Saunders MJ, Tovar MA, Datta S, et al. Pragmatic tuberculosis prevention policies for primary care in low- and middle-income countries. Eur Respir J 2018; 51: 1800315 [https://doi.org/ $10.1183 / 13993003.00315-2018]$. 
Our research group, Innovation For Health And Development (IFHAD; www.ifhad.org), recently derived and validated a score to predict tuberculosis disease among contacts aged $\geqslant 15$ years of patients with laboratory-confirmed tuberculosis, including sputum-smear negative tuberculosis [6]. The IFHAD score incorporates readily collectable data at the time of contact investigation on index case, household and contact factors, including exposure intensity. A preliminary online version is freely available (https://jscalc. io/calc/qoLectXm5TiopbbD). Through the derivation of this score, we have shown that tuberculosis disease in adult contacts may be predicted without any laboratory or invasive testing. Importantly, inclusion of TST results in the score provided no additional predictive value. In children, most cases of tuberculosis occur after missed opportunities for prevention. Although the reasons for this are complex, a key barrier is the perceived necessity to base diagnostic and preventive treatment decisions on TST results. A recent study of 1718 child contacts aged $<16$ years demonstrated that a single TST result does not provide any additional predictive value for tuberculosis disease following documented household exposure [7]. Indeed, the technical and operational limitations of testing for tuberculosis infection in LMICs are well described [8] and further demonstrated in the study by ACUÑA-Villaorduña et al. [4]: 20\% of recruited contacts had incomplete information on TST/IGRA results, and, of those with complete results, $19 \%$ had discordance between the two tests.

As part of the PREVENT TB study [9, 10], our research group is evaluating a policy of risk-based preventive treatment for contacts in Peru, whereby all children aged $<15$ years are recommended to take preventive treatment, irrespective of TST results, and adults are informed of their risk using the IFHAD score. We aim to substantially increase uptake of preventive treatment among contacts and, through doing so, demonstrate that simple policies can be effectively delivered in primary care to prevent tuberculosis in people at high risk.

Matthew J. Saunders $\oplus^{1,2,3,4}$, Marco A. Tovar ${ }^{1,2,3,4}$, Sumona Datta ${ }^{1,2,3,4}$, Benjamin E.W. Evans $s^{3,4}$, Tom Wingfield ${ }^{4,5,6,7}$ and Carlton A. Evans $s^{1,2,3,4}$

${ }^{1}$ Infectious Diseases and Immunity, Imperial College London, London, UK. ${ }^{2}$ Wellcome Trust Imperial College Centre for Global Health Research, London, UK. ${ }^{3}$ Innovation For Health And Development (IFHAD), Laboratory of Research and Development, Universidad Peruana Cayetano Heredia, Lima, Peru. ${ }^{4}$ Innovación Por la Salud Y Desarrollo (IPSYD), Asociación Benéfica PRISMA, Lima, Peru. ${ }^{5}$ Clinical Infection, Microbiology, and Immunity, Institute of Infection and Global Health, University of Liverpool, Liverpool, UK. ${ }^{6}$ Social Medicine, Infectious Diseases and Migration Group, Dept of Public Health Science, Karolinksa Institutet, Stockholm, Sweden. ${ }^{7}$ LIV-TB, Liverpool School of Tropical Medicine, Liverpool, UK.

Correspondence: Matthew J. Saunders, Section of Infectious Diseases and Immunity, Imperial College London Hammersmith Hospital Campus, Commonwealth Building level 8, 150 Du Cane Road, London, W12 0NN, UK.

E-mail: matthew.saunders@ifhad.org

Received: Feb 122018 | Accepted after revision: Feb 142018

Conflict of interest: None declared.

Support statement: Funding was received from the Wellcome Trust (awards 057434/Z/99/Z, 070005/Z/02/Z, 078340/Z/ 05/Z, 105788/Z/14/Z and 201251/Z/16/Z); DFID-CSCF; the Joint Global Health Trials consortium (MRC, DFID, and Wellcome Trust award MR/K007467/1); the STOP TB partnership's TB REACH initiative funded by the Government of Canada and the Bill and Melinda Gates Foundation (awards W5_PER_CDT1_PRISMA and OPP1118545); and the charity IFHAD: Innovation For Health And Development.

\section{References}

1 World Health Organization. Global Tuberculosis Report 2017. WHO/HTM/TB/2017.23. Geneva, World Health Organization, 2017. Available from: www.who.int/tb/publications/global_report/en/

2 Alsdurf H, Hill PC, Matteelli A, et al. The cascade of care in diagnosis and treatment of latent tuberculosis infection: a systematic review and meta-analysis. Lancet Infect Dis 2016; 16: 1269-1278.

3 Lönnroth K, Migliori GB, Abubakar I, et al. Towards tuberculosis elimination: an action framework for low-incidence countries. Eur Respir J 2015; 45: 928-952.

4 Acuña-Villaorduña C, Jones-López EC, Fregona G, et al. Intensity of exposure to pulmonary tuberculosis determines risk of tuberculosis infection and disease. Eur Respir J 2018; 51: 1701578.

5 Mandalakas AM, Kirchner HL, Lombard C, et al. Well-quantified tuberculosis exposure is a reliable surrogate measure of tuberculosis infection. Int J Tuberc Lung Dis 2012; 16: 1033-1039.

6 Saunders MJ, Wingfield T, Tovar MA, et al. A score to predict and stratify risk of tuberculosis in adult contacts of tuberculosis index cases: a prospective derivation and external validation cohort study. Lancet Infect Dis 2017; 17: 1190-1199.

7 Martinez L, Shen Y, Handel A, et al. Effectiveness of WHO's pragmatic screening algorithm for child contacts of tuberculosis cases in resource-constrained settings: a prospective cohort study in Uganda. Lancet Respir Med 2017; in press [https://doi.org/10.1016/S2213-2600(17)30497-6].

8 Rangaka MX, Wilkinson KA, Glynn JR, et al. Predictive value of interferon- $\gamma$ release assays for incident active tuberculosis: a systematic review and meta-analysis. Lancet Infect Dis 2012; 12: 45-55. 
9 BioMed Central Ltd. ISRCTN Registry: ISRCTN17820976. PREVENT TB: Improving determinants of TB cure, prevention \& diagnosis. https://doi.org/10.1186/ISRCTN17820976 Date last updated: October 19, 2016. Date last accessed: February 6, 2018.

10 Wingfield T, Tovar MA, Huff D, et al. A randomized controlled study of socioeconomic support to enhance tuberculosis prevention and treatment, Peru. Bull World Health Organ 2017; 95: 270-280.

Copyright @ERS 2018 